
\title{
$\begin{array}{ll}\text { Research Square } & \begin{array}{l}\text { Preprints are preliminary reports that have not undergone peer review. } \\ \text { They should not be considered conclusive, used to inform clinical practice, }\end{array} \\ \text { or referenced by the media as validated information. }\end{array}$
}

\section{Research on the Potential Mechanism of Chuanxiong Rhizoma on Treating Diabetic Nephropathy Based on Network Pharmacology}

\section{Shanshan $\mathrm{Hu}$}

Department of Pharmacy, Zhujiang Hospital, Southern Medical University, Guangzhou 510282, China

\section{Siteng Chen}

Department of Urology, Shanghai General Hospital, Shanghai Jiao Tong University School of Medicine, Shanghai 200080, China

\section{Zhilei Li}

Department of Pharmacy, Zhujiang Hospital, Southern Medical University, Guangzhou 510282, China

\section{Yuhang Wang}

Department of Pharmacy, Zhujiang Hospital, Southern Medical University, Guangzhou 510282, China

\section{Yong Wang ( $\nabla$ wy43555@smu.edu.cn )}

Department of Pharmacy, Zhujiang Hospital, Southern Medical University, Guangzhou 510282, China

\section{Research}

Keywords: Diabetic Nephropathy, Chuanxiong Rhizoma, Network Pharmacology

Posted Date: April 8th, 2020

DOI: https://doi.org/10.21203/rs.3.rs-18492/v1

License: (c) (1) This work is licensed under a Creative Commons Attribution 4.0 International License. Read Full License

Version of Record: A version of this preprint was published at International Journal of Medical Sciences on January 1st, 2020. See the published version at https://doi.org/10.7150/ijms.47555. 


\section{Abstract}

Background: Chuanxiong Rhizoma is one of the traditional Chinese medicines which have been used for years in the treatment of diabetic nephropathy (DN). However, the mechanism of Chuanxiong Rhizoma in DN has not yet been fully understood.

Methods: We performed network pharmacology to construct targeted proteins interaction network of Chuanxiong Rhizoma. Active ingredients were acquired from the Traditional Chinese Medicine Systems Pharmacology Database and Analysis Platform. DRUGBANK database was used to predict targeted proteins of Chuanxiong Rhizoma. Gene ontology (GO) biological process analyses and KEGG pathway enrichment analyses were also performed for functional prediction of the targeted proteins. Molecular docking was applied for evaluating the drug interactions between hub targets and active ingredients.

Results: Twenty-eight targets fished by 6 active ingredients of Chuanxiong Rhizoma were obtained in the study. The top 10 significant GO analyses, as well as 6 KEGG pathways, were enriched for genomic analysis. We also acquired 1366 differentially expressed genes associated with DN from GSE30528 dataset, including five targeted genes: $\mathrm{KCNH} 2, \mathrm{NCOA} 1, \mathrm{KDR}, \mathrm{NR} 3 \mathrm{C} 2$ and ADRB2. Molecular docking analysis successfully combined KCNH2, NCOA1, KDR and ADRB2 to Myricanone with docking scores from 4.61 to 6.28 . NR3C2 also displayed good binding forces (8.13 and 8.34) with Wallichilide and Sitosterol, revealing good binding forces to active compounds of Chuanxiong Rhizoma.

Conclusions: Chuanxiong Rhizoma might take part in the treatment of DN through pathways associated with steroid hormone, estrogen, thyroid hormone and IL-17. KCNH2, NCOA1, KDR, ADRB2 and NR3C2 were proved to be the hub targets closely related to corresponding active ingredients of Chuanxiong Rhizoma.

\section{Background}

Diabetic nephropathy (DN) is a common microvascular complication of diabetes mellitus (DM), which is also the most chronic complication of DM with high risk of disability and difficulty in curing [1]. About $20 \% \sim 40 \%$ of diabetic patients are accompanied with DN [2], who suffer from 6 times of risk of developing into end-stage renal disease (ESRD) [3]. Although many therapies have been tested in animal models, there is still a lack of effective therapeutic drugs for DN since the pathogenesis of DN is very complex, which has not yet been fully understood.

Traditional Chinese medicine (TCM) has been used for years in the treatment of DN [4]. Chuanxiong Rhizoma is one of these TCM which was usually used in the treatment of brain and heart diseases [5] in the past. Isolated from the alkaloid extracted from Chuanxiong Rhizoma, tetramethylpyrazine has multiple pharmacological effects including anti-oxidation, improving microcirculation, and inhibition the production of glycation [6]. Current studies have also revealed that Chuanxiong Rhizoma could inhibit endothelial cell damage [7], as well as vascular smooth muscle cell proliferation [8]. Chuanxiong Rhizoma could also reduce IL-6, IL-8 and TNF-a level in serum to reduce inflammation [9]. What's more, Chuanxiong 
is a frequently prescribed component in many TCM formulas for the therapy of DN[10][11]. Some researchers have shown that Chuanxiong injection can provide protective effect in patients with DN[12], improve renal function (blood urea nitrogen [BUN] and serum creatinine [SCr]) and reduce in urine protein[13]. However, the mechanism of Chuanxiong Rhizoma in DN has not yet been fully studied.

Using complex network and visualization technology, network pharmacology can combine with TCM to treat diseases with multiple targets, multiple pathways and multi-target synergy, which can provide new ideas and effective measures for TCM mechanism research. In this study, we performed a network pharmacology research focus on Chuanxiong Rhizoma to further explore the possible mechanism of its action on DN, and provide a solid theoretical basis for the follow-up study.

\section{Methods And Materials}

\subsection{Active ingredients acquiring and targeted protein predicting}

Based on Traditional Chinese Medicine Systems Pharmacology Database and Analysis Platform (TCMSP, http://tcmspw.com/tcmsp.php) [14] and DRUGBANK database(https://www.drugbank.ca/) [15], we acquired active ingredients of Chuanxiong Rhizoma by according to the suggested drug screening criteria: oral bioavailability $(O B) \geq 30 \%$ and drug-likeness $(D L) \geq 0.18$. The DRUGBANK database provides accurate and reliable prediction of the target proteins corresponding to chemical small molecules of active ingredients. Gene symbol associated with target proteins could be acquired from the uniprot database(https://www.uniprot.org/) [16]. The action targets related with DN were also screened from the comparative toxicogenomics database (CTD)( http://ctdbase.org/) [17]. And then common targets for Chuanxiong Rhizoma and DN were obtained using Venn diagram. The flow diagram of this study was shown in Fig. 1.

\subsection{Construction of targeted proteins interaction network}

The STRING database (https://string-db.org/cgi/input.pl? sessionld=FE22IrLmPAsy\&input_page_show_search=on) was used to analyze the targeted proteins interactions. The minimum required interaction score was set at 0.4 for network diagram. And then targeted proteins interaction network was constructed using Cytoscape 3.5.1 software (http://www.cytoscape.org) [18].

\subsection{Functional prediction of targeted proteins of Chuanxiong Rhizoma}

Based on the interactions of targeted proteins, we performed Gene ontology (G0) biological process analyses and KEGG pathway enrichment analyses to predict the potential function of targeted proteins of 
Chuanxiong Rhizoma. The clusterProfiler package in R was used for statistical analysis and visualization of functional profiles for genes and gene clusters [19].

\subsection{Hub proteins designation for DN treated with Chuanxiong Rhizoma}

Raw gene data and corresponding clinical data of microarray profile from GSE30528 dataset [20] using the Human Genome U133A 2.0 Array were acquired from Gene Expression Omnibus database (https://www.ncbi.nlm.nih.gov/gds/), and then background adjusted by Robust Multichip Average [21]. GSE30528 dataset includes microarray data of 9 DN glomeruli tissues and 13 controlled normal glomeruli tissues. The limma package [22] available from the Bioconductor project was performed to find out differentially expressed genes (DEGs) between DN glomeruli tissues and controlled normal glomeruli tissues. Absolute value of fold change of gene signature more than 1.5 and $P$ value less than 0.05 were set as the significance threshold. The targeted proteins associated with DEGs of diabetic nephropathy would be selected as hub proteins for subsequent docking analysis.

\subsection{Molecular docking of hub proteins}

In order to evaluate the drug interactions with potential protein targets involved in this research, the selected hub proteins were evaluated by SystemsDock with the high-precision docking simulation assessing protein-ligand interaction [23]. The molecular formulas of active ingredients were obtained from the TCMSP database. Structure files of targeted proteins were acquired from RCSB Protein Data Bank (PDB database, http://www.pdb.org/pdb/home/home.do) [17]. Docking score, ranging from 0 to 10 representing weak combining ability to strong combining ability, represents a negative logarithm of experimental dissociation/inhibition constant value (pKd/pKi) [24].

\subsection{Statistical analysis}

R 3.6.1 (www.rproject.org) and SPSS 13.0 (SPSS Inc., Chicago, IL, USA) were used for data analysis. $P$ value less than 0.05 was considered significant. We also applied Cytoscape 3.5.1 software to visualize the compound-target-pathway interaction network of Chuanxiong Rhizoma in this study.

\section{Results}

\subsection{Active ingredients and targeted protein of Chuanxiong Rhizoma}

Seven active ingredients of Chuanxiong Rhizoma (Mandenol, Myricanone, Perlolyrine, Senkyunone, Wallichilide, Sitosterol and FA) were acquired from TCMSP database according to the suggested screening criteria: $O B \geq 30 \%$ and $\mathrm{DL} \geq 0.18$ (Table 1). One active ingredient (Senkyunone) without any corresponding target was excluded. Based on the reliable prediction of the target protein from the DRUGBANK database, we finally obtained 28 targetd proteins of Chuanxiong Rhizoma after excluding the repeated targets (Table 2). Compound-target network of Chuanxiong Rhizoma was shown in Fig. 2. 
We searched "diabetic nephropathy" in the CTD database and found 18652 DN-related genes. Ultimately, Venn diagram summarized 28 common targets both associated with DN and Chuanxiong Rhizoma (Fig. 3a) for further analysis. Targeted protein interaction network of Chuanxiong Rhizoma from STRING was shown in Fig. $3 b$.

Table 1

Characteristics of the seven active ingredients of Chuanxiong Rhizoma

\begin{tabular}{|lllll|}
\hline Molecule ID & Molecule name & Molecular weight & OB (\%) & DL \\
\hline MOL000433 & FA & 441.45 & 68.96 & 0.71 \\
\hline MOL002140 & Perlolyrine & 264.3 & 65.95 & 0.27 \\
MOL002151 & Senkyunone & 326.52 & 47.66 & 0.24 \\
\hline MOL002157 & Wallichilide & 412.57 & 42.31 & 0.71 \\
MOL001494 & Mandenol & 308.56 & 42 & 0.19 \\
MOL002135 & Myricanone & 356.45 & 40.6 & 0.51 \\
\hline MOL000359 & Sitosterol & 414.79 & 36.91 & 0.75 \\
\hline OB: oral bioavailability; DL: drug-likeness. & & \\
\hline
\end{tabular}

\subsection{GO biological process analyses and KEGG pathway enrichment analyses}

Twenty-eight targets fished by 6 active ingredients of Chuanxiong Rhizoma and DN from CTD database were further analyzed for functional prediction. The top 10 significant $\mathrm{GO}$ analyses, as well as $6 \mathrm{KEGG}$ pathways, were enriched for genomic analysis. In GO biological processes analysis (Fig. $3 \mathrm{c}$ ), the targets were mainly enriched in response to steroid hormone (G0:0048545); rhythmic process (G0:0048511); steroid hormone mediated signaling pathway (G0:0043401); cellular 
Table 2

Predicted target from active ingredients

$\begin{array}{lll}\text { Target Name } & \text { UniProt Gene code General Function } \\ & \text { ID } & \end{array}$

Nitric oxide synthase, inducible

Prostaglandin $\mathrm{G} / \mathrm{H}$ synthase 1

Potassium voltage-gated $\quad$ Q12809 $\quad \mathrm{KCNH} 2$ channel subfamily $\mathrm{H}$ member 2

Estrogen receptor

Androgen receptor

Sodium channel protein

type 5 subunit alpha

Peroxisome proliferator activated receptor gamma

Prostaglandin G/H synthase 2

Coagulation factor VII

Vascular endothelial growth factor receptor 2

Retinoic acid receptor RXRalpha

CGMP-inhibited 3',5'-cyclic phosphodiesterase A

Beta-2 adrenergic recepto

Estrogen receptor beta

Dipeptidyl peptidase IV

Mitogen-activated protein

kinase 14

Glycogen synthase kinase3 beta

Heat shock protein HSP 90

Serine/threonine-protein kinase Chk1
P35228 NOS2 Tetrahydrobiopterin binding

P23219 PTGS1 Prostaglandin-endoperoxide synthase activity

Voltage-gated potassium channel activity involved in ventricular cardiac muscle cell action potential repolarization

Zinc ion binding

Zinc ion binding

Q14524 SCN5A Voltage-gated sodium channel activity involved in sa node cell action potential

P37231 PPARG Zinc ion binding

P35354 PTGS2 Prostaglandin-endoperoxide synthase activity

P08709 F7 Serine-type peptidase activity

P35968 KDR Vascular endothelial growth factor receptor binding

Zinc ion binding

Metal ion binding

Protein homodimerization activity

Zinc ion binding

095718 ESRRB Zinc ion binding

P27487 DPP4 Virus receptor activity

Q16539 MAPK14 Protein serine/threonine kinase activity

P49841 GSK3B Ubiquitin protein ligase binding

P07900 HSP90AB1 Tpr domain binding

014757 CHEK1 Protein serine/threonine kinase activity 


\begin{tabular}{|c|c|c|c|}
\hline Target Name & $\begin{array}{l}\text { UniProt } \\
\text { ID }\end{array}$ & Gene code & General Function \\
\hline Ig gamma- 1 chain $\mathrm{C}$ region & P01857 & IGHG1 & Immunoglobulin receptor binding \\
\hline $\begin{array}{l}\text { Proto-oncogene } \\
\text { serine/threonine-protein } \\
\text { kinase Pim-1 }\end{array}$ & P11309 & PIM1 & Transcription factor binding \\
\hline Cyclin-A2 & P20248 & CCNA2 & $\begin{array}{l}\text { Essential for the control of the cell cycle at } \\
\text { the G1/S (start) and the G2/M (mitosis) } \\
\text { transitions }\end{array}$ \\
\hline $\begin{array}{l}\text { Nuclear receptor } \\
\text { coactivator } 1\end{array}$ & Q15788 & NCOA1 & Transcription coactivator activity \\
\hline $\begin{array}{l}\text { Nuclear receptor } \\
\text { coactivator } 2\end{array}$ & Q15596 & NCOA2 & Transcription coactivator activity \\
\hline Mineralocorticoid receptor & P08235 & NR3C2 & Zinc ion binding \\
\hline Glucocorticoid receptor & P04150 & NR3C1 & Zinc ion binding \\
\hline $\begin{array}{l}\text { Cell division protein kinase } \\
2\end{array}$ & P24941 & CDK2 & Metal ion binding \\
\hline Progesterone receptor & P06401 & PGR & Zinc ion binding \\
\hline
\end{tabular}

response to drug (G0:0035690); hormone-mediated signaling pathway (GO:0009755); cellular response to steroid hormone stimulus (G0:0071383); reproductive structure development (G0:0048608); intracellular receptor signaling pathway (G0:0030522); transcription initiation from RNA polymerase II promoter (GO:0006367); DNA-templated transcription, initiation (G0:0006352). Figure 3d reveals the enriched KEGG pathways of the targets, including progesterone-mediated oocyte maturation (hsa04914), thyroid hormone signaling pathway (hsa04919), estrogen signaling pathway (hsa04915), IL-17 signaling pathway (hsa04657), small cell lung cancer (hsa05222) and prostate cancer (hsa05215).

\subsection{Construction of compound-target protein-pathway network of Chuanxiong Rhizoma}

Compound-target protein-pathway network of Chuanxiong Rhizoma was constructed using Cytoscape 3.5.1 software. The interaction network was constituted by 6 active ingredients of Chuanxiong Rhizoma, 28 corresponding targeted protein (shown by gene symbol), 10 gene ontology biological process and 6 KEGG pathways. As shown in Fig. 4, squires represent active compounds of Chuanxiong Rhizoma, rotundities represent gene symbols of targets, hexagons represent enriched $\mathrm{GO}$ biological processes and diamonds represent enriched KEGG pathways.

\subsection{Hub proteins designation and molecular docking}


Based on GSE30528 dataset, 1366 DEGs associated with DN were found according to the significance threshold: absolute value of fold change of gene signature more than 1.5 and $P$ value less than 0.05 . 1366 DEGs were shown as green plots in Fig. 5a, including five targeted genes: KCNH2, NCOA1, KDR, NR3C2 and ADRB2 (red plots in Fig. 5a). Further analysis revealed that gene signatures of KCNH2, NCOA1, KDR and NR3C2 were significantly lower in DN glomeruli tissues compared to controlled normal glomeruli tissues, while gene signature of ADRB2 was significantly higher in DN glomeruli tissues (Fig. 5b). The corresponding five targeted proteins were designed as hub proteins with ligand-protein interaction diagrams (Fig. 5c) for further molecular docking analysis.

With the help of SystemsDock [23], we successfully combined KCNH2, NCOA1, KDR and ADRB2 to Myricanone with docking scores from 4.61 to 6.28 . What more, NR3C2 also displayed good binding forces (8.13 and 8.34) with Wallichilide and Sitosterol (Fig. 5d), revealing good binding forces to active compounds of Chuanxiong Rhizoma.

\section{Discussion}

As one of the principal causes of ESRD around the world, DN is closely related to cardiovascular and cerebrovascular diseases and increased mortality of DM patients [25]. DN is characterized by proteinuria and changes in renal ultrastructure, and the prognosis of patients with DN is poor, especially for these patients with ESRD. It takes place approximately a quarter of patients with type $2 \mathrm{DM}$ and about $33 \%$ of type 1 DM patients [26][27]. Due to the limitations in the understanding of DN, currently, there is still a lack of accurate treatment strategies specifically targeting at DN in addition to controlling blood sugar, blood lipid levels and hypertension.

In this study, we carried out a network pharmacology research focus on Chuanxiong Rhizoma, one of the important TCM used in treating DN, to further explore its possible mechanism on DN. By applying GO biological process analyses, the special targets of Chuanxiong Rhizoma were significantly enriched in hormone-mediated signaling pathway, response to steroid hormone, and other pathways related to reproductive structure development. Previous studies have suggested that steroid hormone might play a vital role in the pathogenesis of DN in DM patients [28][29][30]. Estradiol could inhibit the transcription of type IV collagen and reduce the expression of collagen by way of activating tyrosine kinase 2 and inhibiting the synthesis of TGF- $\beta$, so as to alleviate fibrosis in DN patients [31]. Our study revealed that Chuanxiong Rhizoma might target at steroid hormone in the treatment of DN.

KEGG pathway enrichment analyses suggested that Chuanxiong Rhizoma was associated with estrogen signaling pathway, thyroid hormone signaling pathway and IL-17 signaling pathway in the treatment of DN. Estrogen has been reported to act on renal protection by up-regulating the level of endothelial NO synthase and inhibiting the synthesis of inducible NO synthase to maintain normal renal function [32] [33]. DN patients are often accompanied by hypothyroidism, and even low triiodothyronine syndrome. Serum free triiodothyronine acts as a key predictor of prognosis in patients with DN [34]. In addition, thyroid hormone replacement therapy could reduce the risk of cardiovascular diseases in DN patients 
with hypothyroidism [35]. Recently, the relationship between IL-17 and DN has attracted more and more attentions from the researchers. Patients with DN are reported to have elevated levels of IL-17 in their peripheral blood [36]. Animal study showed that activation of ThI7 and increase of IL-17 expression play important roles in the pathogenesis of DN [37]. Here, with the help of network pharmacology analysis, our study revealed that Chuanxiong Rhizoma might take part in the treatment of DN through pathways associated with estrogen, thyroid hormone and IL-17.

GSE30528 dataset [19] contains microarray data of 9 DN glomeruli tissues and 13 controlled normal glomeruli tissues. By setting the significance threshold of absolute value of fold change of gene signature $>1.5$ and $P$ value $<0.05,1366$ DEGs associated with DN were obtained, including five hub genes/proteins targeted by Chuanxiong Rhizoma. In our study, KCNH2, NCOA1, KDR, ADRB2 and NR3C2 are closely related to corresponding active ingredients of Chuanxiong Rhizoma with favorable molecular docking scores, indicating good binding relationships between compounds and targeted proteins. Among the hub targets, $\mathrm{KCNH} 2$ is involved in ventricular cardiac muscle cell action potential repolarization, while NCOA1 is enriched in thyroid hormone signaling pathway and estrogen signaling pathway. ADRB2 regulates beta-2 adrenergic receptor ( $\beta 2 A R)$, which has been reported to act on inhibiting of macrophage function [38] and be involved in LPS-induced activation of THP-1 cells [39]. Agonists of $\beta 2 A R$ are involved in the regulation of macrophage activation in diabetic cardiovascular and renal complications [40].

There are still some shortcomings in our study. Firstly, we just explored the potential functional mechanism of Chuanxiong Rhizoma on DN, without analyzing the mutual interferences of drug components. Secondly, this study failed to perform stratification analysis of different pathological stages of DN. Finally, the key targets and pathways we obtained here have not been experimentally verified. Further molecular mechanism researches and clinical validations will be carried out in our future work.

\section{Conclusions}

Chuanxiong Rhizoma has been proven to play a role in the treatment of DN. Through network pharmacology analysis, our study revealed that Chuanxiong Rhizoma might take part in the treatment of DN through pathways associated with steroid hormone, estrogen, thyroid hormone and IL-17. Molecular docking supported the application of KCNH2, NCOA1, KDR, ADRB2 and NR3C2, which were closely related to corresponding active ingredients of Chuanxiong Rhizoma. However, further molecular mechanism researches and clinical validations are still needed in the future.

\section{Abbreviations}

DN: diabetic nephropathy; GO: Gene Ontology; DM; diabetes mellitus; ESRD: end-stage renal disease; TCM: Traditional Chinese Medicine; BUN: blood urea nitrogen; SCr: serum creatinine; TCMSP: Traditional Chinese Medicine Systems Pharmacology Database and Analysis Platform; OB: oral bioavailability; DL: drug-likeness; CTD: comparative toxicogenomics database; KEGG: Kyoto Encyclopedia of Genes and Genomes; DEGs: differentially expressed genes; PDB: Protein Data Bank. 


\section{Declarations}

\section{Ethics approval and consent to participate}

Not applicable.

\section{Consent for publication}

The manuscript is approved by all authors for publication.

\section{Availability of data and materials}

The data and materials generated or analyzed during this study are available from the corresponding author on reasonable request.

\section{Competing interests}

The authors declare that they have no competing interests.

\section{Funding}

This work was supported by the National Natural Science Foundation of China (Grant numbers 81302738).

\section{Authors' contributions}

H-SS and C-ST conceived and designed the research methods. L-ZL and W-YH collected the data. C-ST analyzed the data. H-SS wrote the paper. H-SS and C-ST contributed equally to this work. W-Y supervised this research. All authors read and approved the final manuscript.

\section{Acknowledgement}

Not applicable.

\section{Authors' information}

H-SS: h1435086126@163.com; C-ST: siteng@sjtu.edu.cn; L-ZL: 15626059979@163.com; W-YH: yuhangwang2019@163.com;W-Y:wy43555@smu.edu.cn.

\section{References}

1. Sung JK, Koh JH, et al. Aldose reductase inhibitor ameliorates renal vascular endothelial growth factor expression in streptozotocin-induced diabetic rats, Yonsei Med. J 2010.

2. Afkarian M, Zelnick LR, et al. Clinical manifestations of kidney disease among US adults with diabetes, JAMA - J. Am. Med. Assoc 2016, 1988-2014. 
3. Rosolowsky ET, Skupien J, et al. Risk for ESRD in type 1 diabetes remains high despite renoprotection, J. Am. Soc. Nephrol 2011.

4. Sun GD, Li CY, et al. Review of Herbal Traditional Chinese Medicine for the Treatment of Diabetic Nephropathy, J. Diabetes Res 2016.

5. Ho J, Jie M, Pharmacological Activity of Cardiovascular Agents from Herbal Medicine, Cardiovasc. Hematol. Agents Med. Chem 2008.

6. Zhao Y, Liu Y, et al. Mechanisms and Clinical Application of Tetramethylpyrazine (an Interesting Natural Compound Isolated from Ligusticum Wallichii): Current Status and Perspective., Oxid. Med. Cell. Longev. 2016 (2016) 2124638.

7. Wang GF, Shi CG, et al. Tetramethylpyrazine attenuates atherosclerosis development and protects endothelial cells from ox-LDL, Cardiovasc. Drugs Ther 2013.

8. Liang MJ, He LC, et al. Screening, analysis and in vitro vasodilatation of effective components from Ligusticum Chuanxiong, Life Sci 2005.

9. Zengyong $Q$, Jiangwei $M$, et al. Effect of Ligusticum wallichii aqueous extract on oxidative injury and immunity activity in myocardial ischemic reperfusion rats, Int. J. Mol. Sci 2011.

10. Song, Yange. Randomized Controlled Study of Chuanxiong Injection combined with Benazepril in the Treatment of Diabetic Nephropathy. J Pract Tradit Chinese Intern Med. 2019;33:13-5.

11. W.H. Zuo Weijuan,Song Beibei et al. Exploration of the Compatibility of Ligusticum Wallichii in the Prevention and Treatment of Diabetic Nephropathy, China J. Chinese Med 2012. 27, 196-198.

12. Yang WJ, Li YR, et al. Protective effect of the ethanol extract from Ligusticum chuanxiong rhizome against streptozotocin-induced diabetic nephropathy in mice, J. Ethnopharmacol. 2018.

13. Wang B, Ni Q, et al. Meta-analysis of the clinical effect of ligustrazine on diabetic nephropathy, Am. J. Chin. Med, 2012.

14. Li Y, Zhang J, et al. Systems pharmacology to decipher the combinational anti-migraine effects of Tianshu formula, J. Ethnopharmacol 2015.

15. Wishart DS, Feunang YD, et al. DrugBank 5.0: A major update to the DrugBank database for 2018, Nucleic Acids Res 2018.

16. Bateman A, Martin MJ, et al. UniProt: The universal protein knowledgebase, Nucleic Acids Res 2017.

17. Berman HM, Westbrook J, et al. The Protein Data Bank (www.rcsb.org), Nucleic Acids Res. 2000.

18. Merico D, Isserlin R, et al. Enrichment map: A network-based method for gene-set enrichment visualization and interpretation, PLoS One 2010.

19. Yu G, Wang LG, et al. ClusterProfiler: An R package for comparing biological themes among gene clusters, Omi. Biol: A J. Integr; 2012.

20. Transcriptome analysis of human diabetic kidney disease Diabetes Woroniecka KI, Park ASD, et al. Transcriptome analysis of human diabetic kidney disease, Diabetes 2011. 
21. Irizarry RA, Bolstad BM, et al. Summaries of Affymetrix GeneChip probe level data, Nucleic Acids Res 2003.

22. Ritchie ME, Phipson B, et al. Limma powers differential expression analyses for RNA-sequencing and microarray studies, Nucleic Acids Res 2015.

23. Hsin KY, Matsuoka Y, et al. systemsDock: a web server for network pharmacology-based prediction and analysis, Nucleic Acids Res 2016.

24. Hsin K-Y, Ghosh S, et al. Combining machine learning systems and multiple docking simulation packages to improve docking prediction reliability for network pharmacology., PLoS One 2013.

25. Duran-Salgado MB, Rubio-Guerra AF. Diabetic nephropathy and inflammation. World J Diabetes. 2014;5:393-8.

26. Sanchez AP, Sharma K. Transcription factors in the pathogenesis of diabetic nephropathy, Expert Rev. Mol. Med 2009.

27. Lei $L$, Mao $Y$, et al. Percentage of circulating CD8 $+T$ lymphocytes is associated with albuminuria in type 2 diabetes mellitus, Exp. Clin. Endocrinol. Diabetes 2014.

28. Maric C, Forsblom C, et al. Association between testosterone, estradiol and sex hormone binding globulin levels in men with type 1 diabetes with nephropathy, Steroids 2010.

29. Fukui M, Soh J, et al. Low serum testosterone concentration in middle-aged men with type 2 diabetes, Endocr. J 2007.

30. Grossmann M, Thomas MC, et al. Low testosterone levels are common and associated with insulin resistance in men with diabetes, J. Clin. Endocrinol. Metab 2008.

31. Zdunek M, Silbiger S, et al. Protein kinase CK2 mediates TGF- $\beta 1$-stimulated type IV collagen gene transcription and its reversal by estradiol, Kidney Int 2001.

32. Xie P, Liu ML, et al. Oestrogen improves glucose metabolism and insulin signal transduction in HepG2 cells, Clin. Exp. Pharmacol. Physiol 2003.

33. Baiardi G, Macova M, et al. Estrogen upregulates renal angiotensin II AT1 and AT2 receptors in the rat, Regul. Pept 2005.

34. Lin Y, Sun Z. Thyroid hormone ameliorates diabetic nephropathy in a mouse model of type II diabetes, J. Endocrinol 2011.

35. Seo C, Kim S, et al. THYROID HORMONE REPLACEMENT REDUCES the RISK of CARDIOVASCULAR DISEASES in DIABETIC NEPHROPATHY PATIENTS with SUBCLINICAL HYPOTHYROIDISM, Endocr. Pract 2018.

36. Zhang C, Xiao C, et al. The alteration of Th1/Th2/Th17/Treg paradigm in patients with type 2 diabetes mellitus: Relationship with diabetic nephropathy. Hum Immunol. 2014;75:289-96.

37. Yu R, Bo H, et al. The Inhibitory Effect of Rapamycin on Toll Like Receptor 4 and Interleukin 17 in the Early Stage of Rat Diabetic Nephropathy. Res: Kidney Blood Press; 2016.

38. Abrass CK, O'Connor SW, et al. Characterization of the $\beta$-adrenergic receptor of the rat peritoneal macrophage, J. Immunol 1985. 
39. Wang W, Zhang Y, et al. Fenoterol inhibits LPS-induced AMPK activation and inflammatory cytokine production through $\beta$-arrestin-2 in THP-1 cell line, Biochem. Biophys. Res. Commun. 2015.

40. Noh H, Yu MR, et al. Beta 2-adrenergic receptor agonists are novel regulators of macrophage activation in diabetic renal and cardiovascular complications, Kidney Int 2017.

\section{Figures}

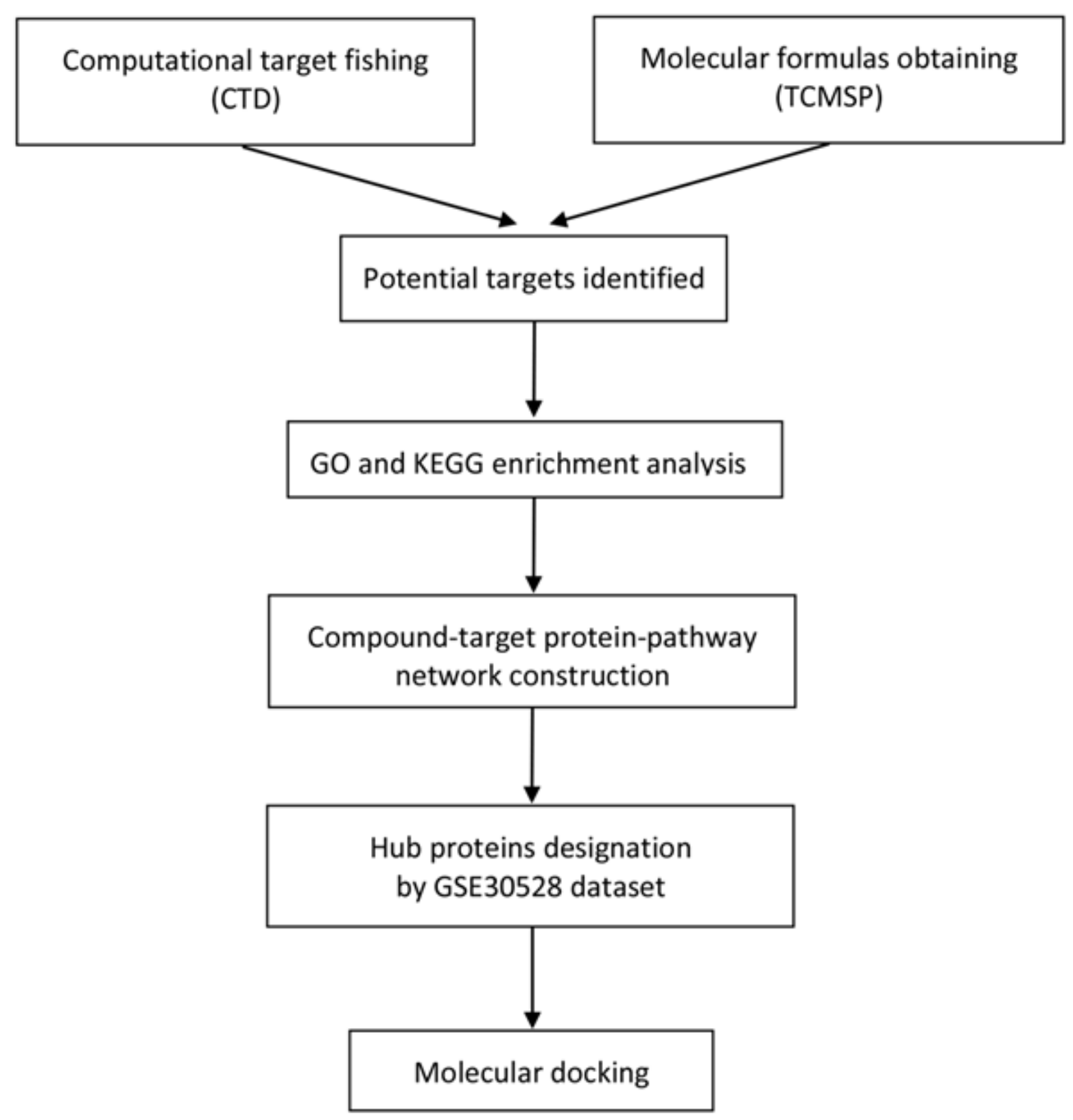

Figure 1

Flow diagram of the research 


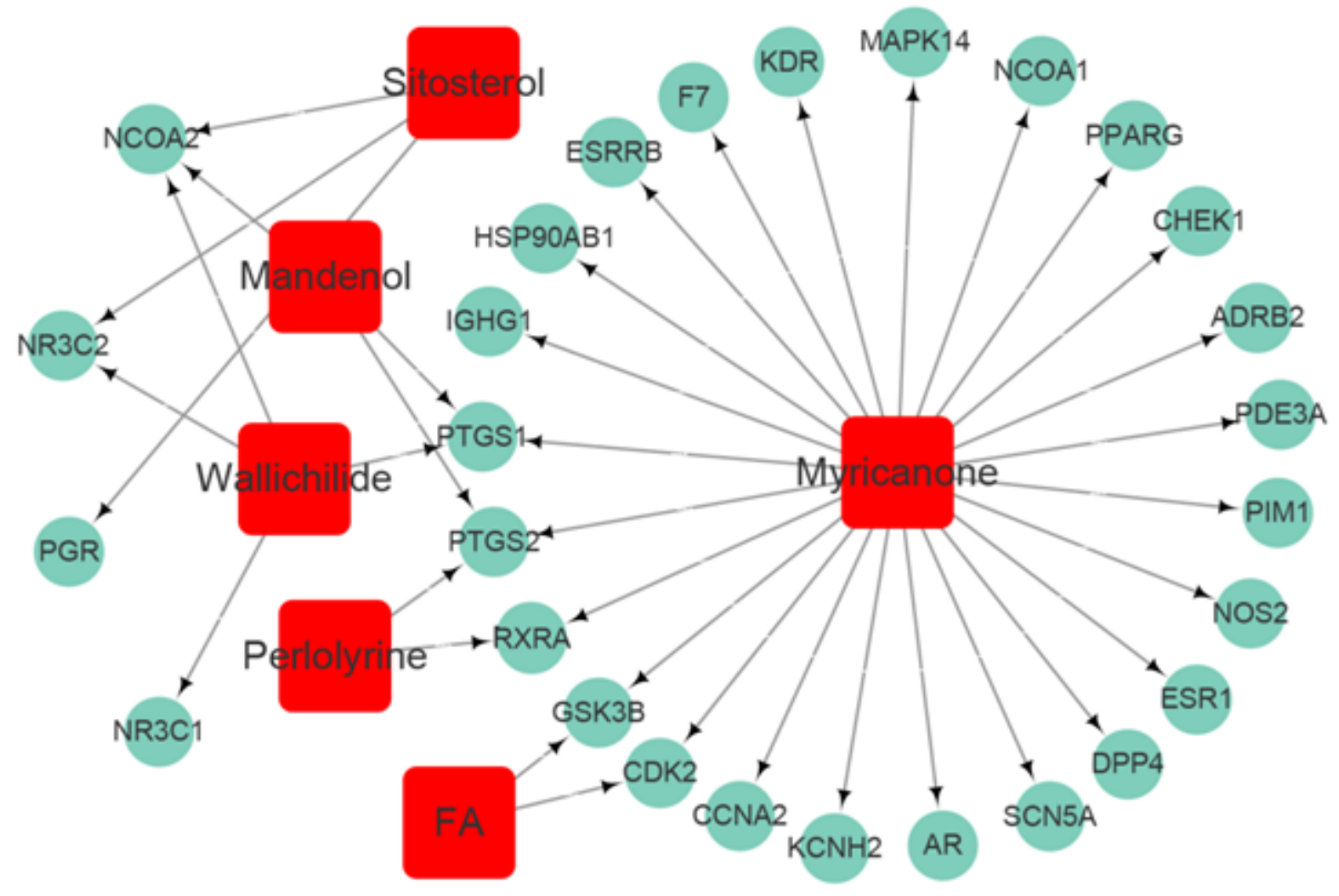

Figure 2

Compound-target network of Chuanxiong Rhizoma. Squires represent active compounds of Chuanxiong Rhizoma. Rotundities represent gene symbols of targets. 
a
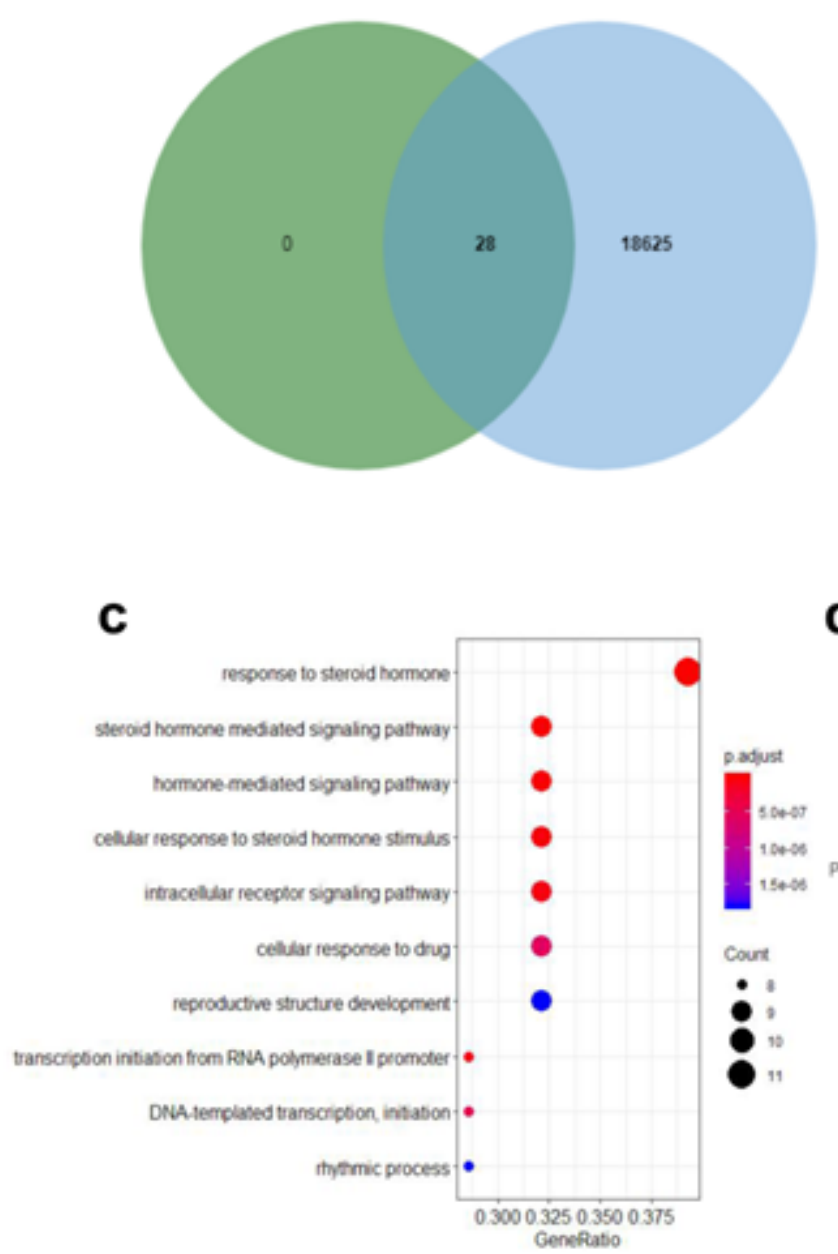

b

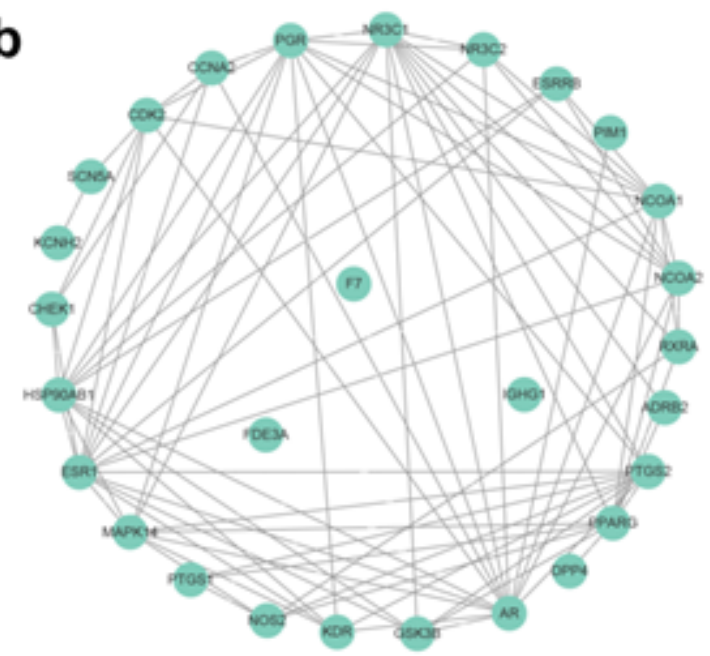

d

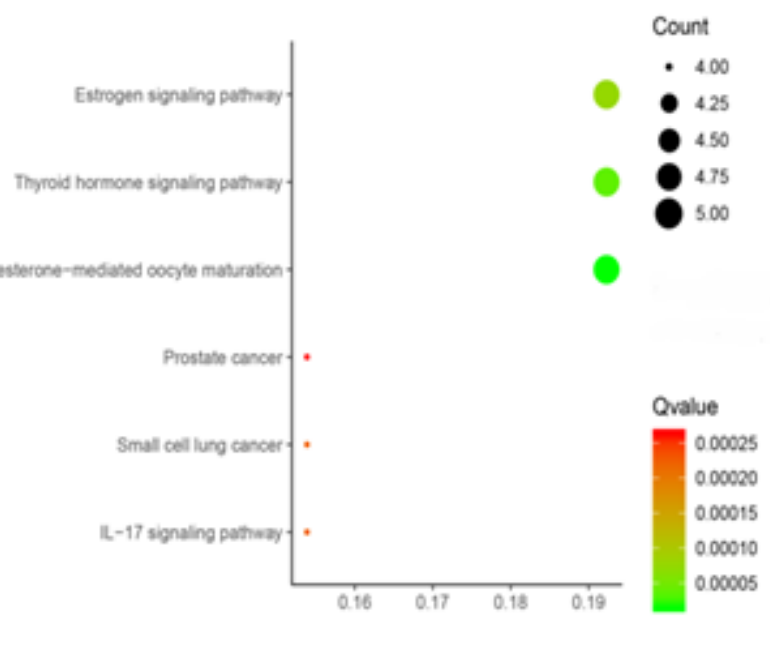

\section{Figure 3}

Venn diagram summaries common targets associated with diabetic nephropathy and Chuanxiong Rhizoma; b. Targeted protein interaction network of Chuanxiong Rhizoma from STRING; c. Gene ontology enrichment analysis of 28 specialized targets; d. KEGG pathway enrichment analysis of 28 specialized targets. 


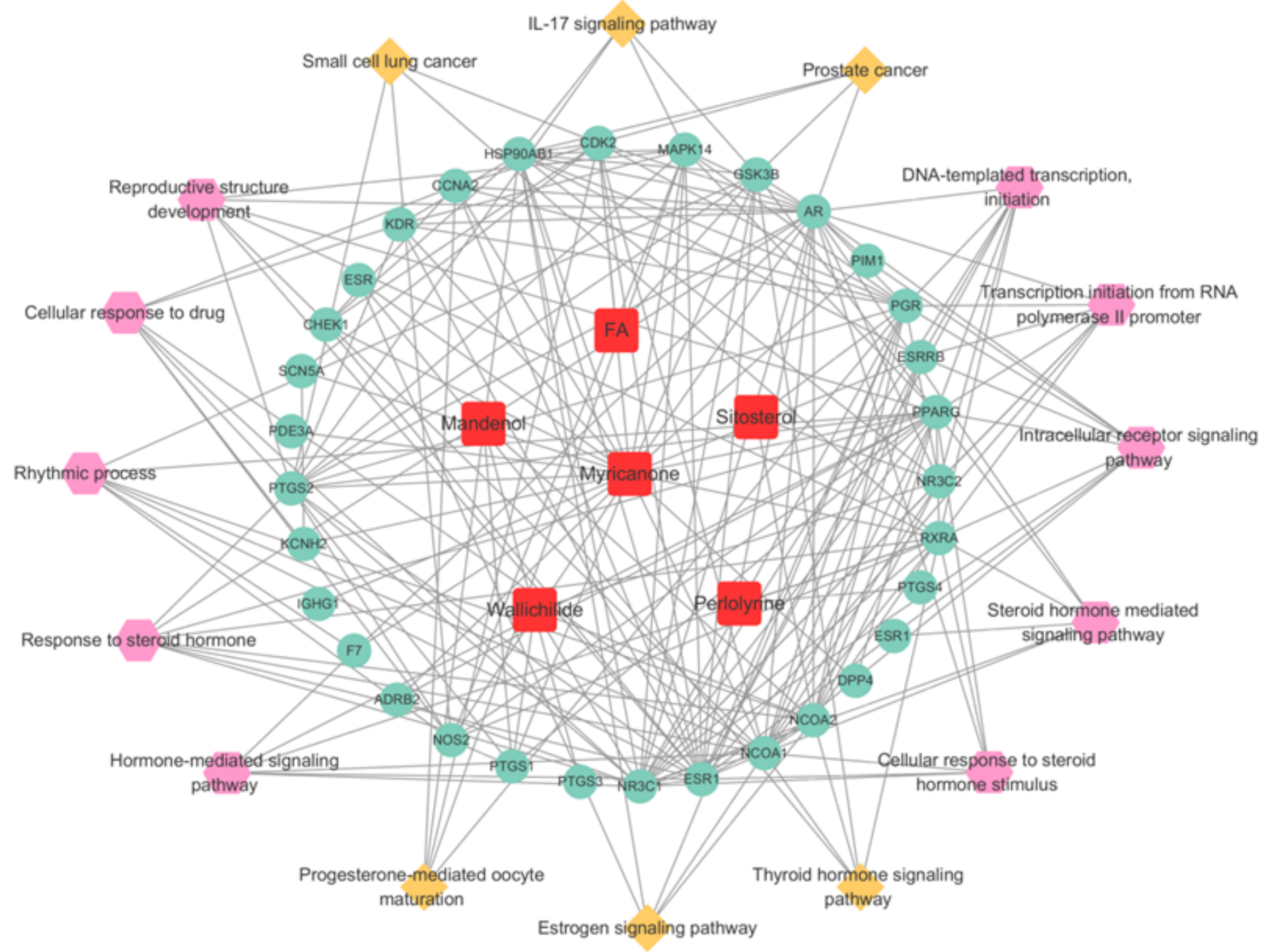

Figure 4

Prediction of compound-target protein-pathway network of Chuanxiong Rhizoma. Squires represent active compounds of Chuanxiong Rhizoma. Rotundities represent gene symbols of targets. Hexagons represent enriched gene ontology biological processes. Diamonds represent enriched KEGG pathways. 

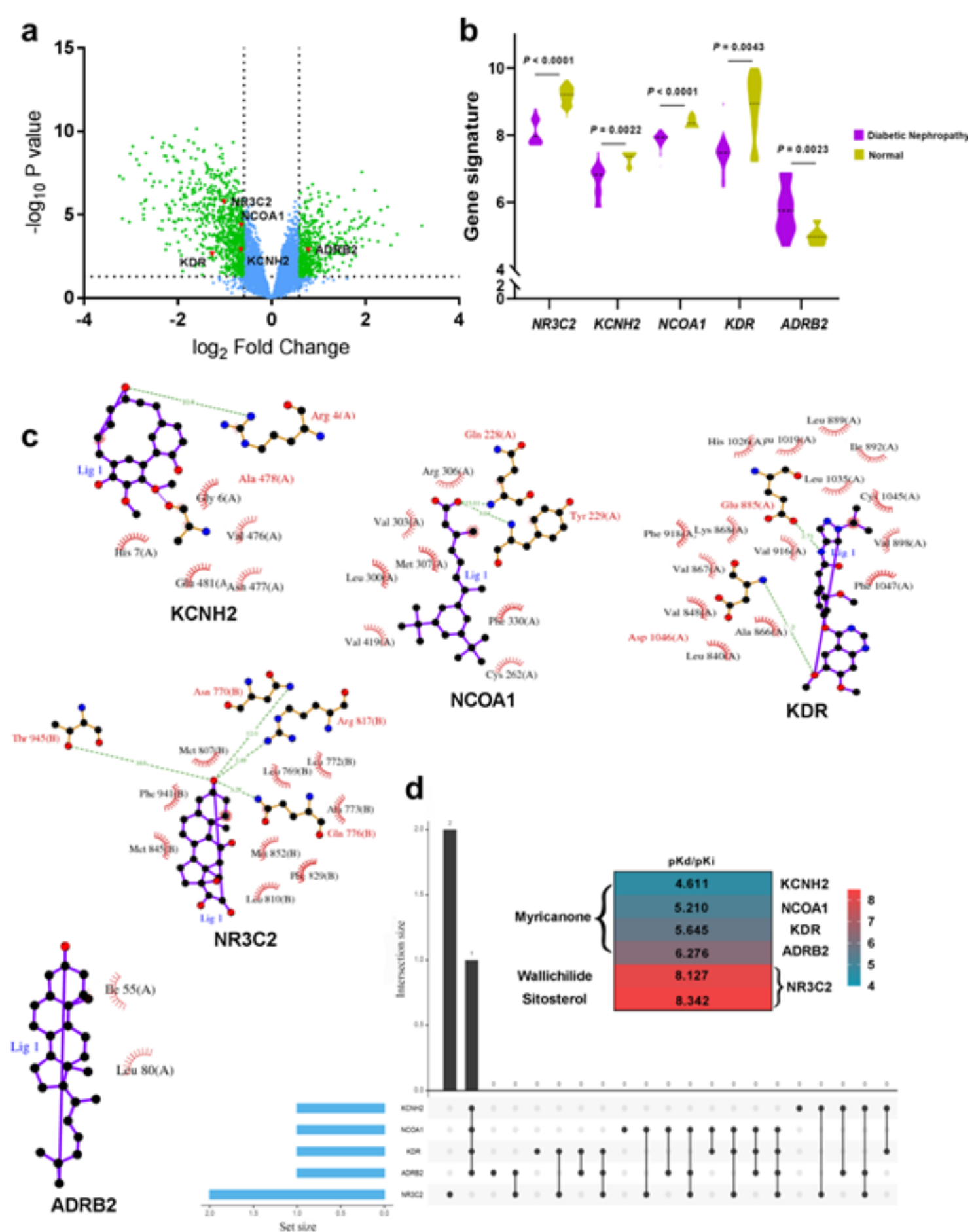

\section{Figure 5}

Volcano Plot represents differentially expressed genes (DEGs) associated with diabetic nephropathy from GSE 30528 dataset and 5 hub proteins (gene symbol: KCNH2, NCOA1, KDR, NR3C2 and ADRB2) targeted by Chuanxiong Rhizoma; green plots represent DEGs; red plots represent hub genes/proteins; $b$. Different gene signatures of hub genes in diabetic nephropathy patients compared with controlled patients in GSE 30528 dataset; c. Ligand-protein interaction diagrams of hub proteins; d. Heatmap shows docking scores (pKd/pKi) of hub proteins combining to active compounds of Chuanxiong Rhizoma. 